General Letters in Mathematics (GLM) 1(1) (2016) 32-38
General Letters in Mathematics (GLM)
Website: hitp://www.sciencereflection.com/general-letters-in-mathematics/
Scfience
Reflection

\title{
The Invariance of the Reverse Order Law under Generalized Inverses of the Product of Two Closed Range Bounded Linear Operators on Hilbert Spaces and Characterization of the Property by the Norm Majorization
}

\author{
Hanifa Zekraoui $^{1}$, Cenap Özel ${ }^{2}$ \\ 1 Faculty of Exact and Natural Sciences, Department of Mathematics \\ University L'Arbi Ben M’hidi BP 358, Oum-El-Bouaghi 04000, Algeria \\ 1 hanifazekraoui@yahoo.fr \\ 2 Faculty of Sciences, Department of MathematicsDokuz Eylul University, Tinaztepe Kampus 35160, Buca, izmir, Turkey, \\ and,Faculty of Sciences, Department of MathematicsKing Abdulaziz University, KAU 21589, Jeddah, Saudi Arabia. \\ 2 cenap.ozel@gmail.com
}

\begin{abstract}
In this paper we extend the invariance of the product $A C^{-} B$ under a generalized inverse $C^{-}$of a matrix $\mathrm{C}$ in finite dimensional vector spaces to the Hilbert spaces by using Douglas's theorem, then we investigate the result and results of the reverse order on Hilbert spaces to study the equivalent conditions for the invariance of the property of the reverse order law for the product of two closed range linear bounded operators on Hilbert spaces. Then, we characterize the property by the norm majorization.
\end{abstract}

\section{Indexing terms/Keywords}

Generalized inverse, Moore-Penrose inverse, reverse order law, norm majorization, factorization, Hilbert space.

\section{SUBJECT CLASSIFICATION}

MSC[2010]: 15A09, 46C07, 46C15, 47A68, 15A23.

\section{Introduction}

If $S$ is a semigroup with unit 1 , and if $a, b \in S$ are invertible, then the equality $(a b)^{-1}=b^{-1} a^{-1}$ is called the reverse order law for the ordinary inverse. It is well-known that the reverse order law does not hold for various classes of generalized inverses. Hence, a significant number of papers treated the equivalent conditions such that the reverse order law holds for the Moore-Penrose inverse of operators on Hilbert or 
Banach spaces(see [5]). Let $A, B$ and $C$ be finite matrices over the real or the complex number field. Based on the conditions for the invariance of the product $A C^{-} B$ under the choice of the generalized inverse $C^{-}$of $C$, equivalent conditions for the invariance of the reverse order of the product $(A B)^{-}=B^{-} A^{-}$of two finite matrices $A$ and $B$ under the choice of their generalized inverses $A^{-}$and $B^{-}$is given [13]. Also, this subject is treated in an other way based on the maximal and minimal rank equations of matrices [12]. This paper consists of two parts. The first part extends the invariance of $A C^{-} B$ in finite dimensional vector spaces to Hilbert spaces, by using Douglas's theorem [7], and investigates this result and known results of the reverse order in Hilbert spaces, to study the equivalent conditions for the invariance of the property of the reverse order law for the product of two closed range bounded linear operators on Hilbert spaces. The second part characterizes the range inclusions conditions for the reverse order of the product of two linear operators under the Moore -Penrose inverse by the norm majorization, using the norms of these operators ending this section by an illustrative example. Let $\mathbb{H}, \mathbb{H}^{\prime}$ be two Hilbert spaces and $\mathbb{B}\left(\mathbb{H}, \mathbb{H}^{\prime}\right)$ denoted the set of all linear bounded operators from $\mathbb{H}$ to $\mathbb{H}^{\prime}$. For $A \in B\left(\mathbb{H}^{\prime} \mathbb{H}^{\prime}\right)$ the null-space and the range of $A$ are denoted by $\mathcal{N}(A)$ and $\mathcal{R}(A)$ respectively. The rank and the trace of $A$ are $r(A)$ and $\operatorname{tra}(A)$. The conjugate transpose of $A$ is denoted by $A^{*}$ and the norm $\|A\|=\left(\operatorname{tra}\left(A^{*} A\right)\right)^{\frac{1}{2}}$. An operator $B \in B\left(\mathbb{H}, \mathbb{H}^{\prime}\right)$ is a generalized inverse of $A$, also it said to be a $\{1\}$-inverse of $A$, if $A B A=A$ holds. In this case $A$ is $\{1\}$-invertible. It is well-known that $A$ is $\{1\}$-invertible if and only if $R(A)$ is closed in $\mathbb{H}^{\prime}$ [4], [6]. The Moore-Penrose inverse of $A \in B\left(\mathbb{H}^{\prime} \mathbb{H}^{\prime}\right)$ is the operator $X \in B\left(\mathbb{H}, \mathbb{H}^{\prime}\right)$ which satisfies the Penrose equations:
(1) $A X A=A$
(2) $X A X=X$
(3) $(A X)^{*}=A X$
(4) $(X A)^{*}=X A$.

The Moore-Penrose inverse of $A$ exists if and only if $\mathcal{R}(A)$ is closed in $\mathbb{H}^{\prime}$ [11]. If the Moore-Penrose inverse of $A$ exists, then it is unique, and it is denoted by $A+$. Recalling that on finite dimensional vector space on $\mathbb{C}$, for any self-adjoint matrix $A$, there exists a unitary matrix $U$ and a diagonal matrix

$D=\operatorname{diag}\left(\lambda_{1}, \ldots, \lambda_{r}, 0, \ldots, 0\right)$, where $\lambda_{i}$ are the eigenvalues of $A$ for $i=1, \ldots, r=r(A)$ such that $A=U * D U$, then the Moore-Penrose inverse $A+=U * \operatorname{diag}\left(\lambda_{1}^{-1}, \ldots, \lambda_{r}^{-1}, 0, \ldots, 0\right) U$. If $A$ is any matrix, then

$A+=(A * A)+A *$. Some algebraic properties related to the generalized inverses $A^{-}$in general and $A+$ in particular are given by the following lemma:

Lemma 1.1 Let $A \in B\left(\mathcal{H}, \mathbb{H}^{\prime}\right)$ with closed range and $A^{-}$be a $\{1\}$-inverse of $A$, then

i) $A A^{-}$and $A^{-} A$ are two bounded linear projectors such that $R\left(A A^{-}\right)=R(A)$ and $N(A) \subset N\left(A^{-} A\right)$. 
ii) If $A^{-}$is a $\{1,2\}$-inverse of $A$, Then $N(A)=N\left(A^{-} A\right)$.

iii) If $A^{-}=A+$, then $N(A+)=N\left(A^{*}\right)$ and $R(A+A)=R(A+)=R\left(A^{*}\right)$.

For more knowledge on the properties of generalized inverses of operators on Hilbert spaces, and the proof of the precedent lemma, see [6], also a short course on generalized inverses of operators on Banach and Hilbert spaces is given in [10].

Lemma 2.1 ([1]) Let $A$ and $B$ be nonzero finites matrices such that $A C^{-} B$ is defined. Then $A C^{-} B$ is invariant under the choice of $C^{-}$a generalized inverse of the matrix $C$, if and only if $R(B) \subset R(C)$ and $R$ $\left(A^{*}\right) \subset R\left(C^{*}\right)$.

Lemma 3.1 (Douglas's Theorem) [7], [8] If A and B are operators on a Hilbert space $\mathbb{Z}$, then the following conditions are equivalent:

(i) $A=B C$ for some operator $C$ on $\mathbb{H}$.

(ii) $\left\|A^{*} x\right\| \leq k\left\|B^{*} x\right\|$ for some $k \geq 0$ and all $x$ in $\mathbb{H}$.

(iii) $R(A) \subset R(B)$.

\section{The invariance of $\mathrm{AC}^{-} \mathrm{B}$ in Hilbert spaces.}

We will extend lemma 2.1 to the bounded linear operators on Hilbert spaces.

Theorem 1.2 Let $A$ and $B$ be two bounded linear operators and $C$ be a closed range bounded linear operator on Hilbert space $H$. Then, $A C^{-} B$ is invariant under the choice of $C^{-}$a generalized inverse of the operator $C$, if and only if $R(B) \subset R(C)$ and $R\left(A^{*}\right) \subset R\left(C^{*}\right)$.

Proof. The if part: let $R(B) \subset R(C)$ and $R\left(A^{*}\right) \subset R\left(C^{*}\right)$, then by Lemma $3, B=C U$ and $A^{*}=C^{*} V^{*}$ for some $U$ and $V^{*}$ operators on $H$. Then $A=V C$. Hence, $A C^{-} B=V C C^{-} C U=V C U$, which is independent from $C^{-}$a $\{1\}$ - inverse of the operator $C$.

Let $B \in B\left(\mathcal{H}, \mathbb{H}^{\prime}\right)$ and $A \in B\left(\mathcal{H}^{\prime}, \mathbb{H}^{\prime \prime}\right)$ be two closed range bounded linear operators. By [4], [6] and [10], the $\{1\}$-inverses and the Moore-Penrose inverses of $A$ and $B$ exist. The reverse order law property for a generalized inverse of the product $A B$ is $(A B)^{-}=B^{-} A^{-}$for $A^{-}$and $B^{-}$are $\{1\}$-inverses of $A$ and $B$ respectively. The main result is to establish the equivalent conditions so that the property holds and be invariant under the choice of $A^{-}$and $B^{-}$. 
Theorem 2.2 Let $B \in B\left(H_{H} \mathbb{H}^{\prime}\right)$ and $A \in B\left(\mathcal{H}^{\prime}, \mathbb{H}^{\prime \prime}\right)$ be two closed range bounded linear operators. The following conditions are equivalent:

i) $A B B^{-} A^{+} A B$ is invariant under the choice of $B^{-}$if and only if $R\left(A^{+} A B\right) \subset R(B)$.

ii) $A B B^{+} A^{-} A B$ is invariant under the choice of $A^{-}$if and only if $R\left(B B^{+} A^{*}\right) \subset R\left(A^{*}\right)$.

iii) $B^{-} A^{-}$is a $\{1\}$-inverse of $A B$ for every $A^{-}$and every $B^{-}\{1\}$-inverses of $A$ and $B$ respectively if and only if $R\left(A^{+} A B\right) \subset R(B)$ and $R\left(B B^{+} A^{*}\right) \subset R\left(A^{*}\right)$.

Proof. It is obviously to show that statements i) and ii) are verified by Theorem 2.1 . We shall prove the third one. The if part: By Lemma 1.1, the first inclusion yields $B B^{+} A^{+} A B=A^{+} A B$.

Multiplying on the left by $A$ gives

$$
A B B^{+} A^{+} A B=A B
$$

By i), for every $B^{-}$a $\{1\}$-inverse of $B$, we have

$$
A B B^{-} A^{+} A B=A B B^{+} A^{+} A B=A B .
$$

From the fact that $R\left(A^{*}\right)=R\left(A^{+}\right)=R\left(A^{+} A\right)$ and the second inclusion, it follows that

$$
A^{+} A B B^{+} A^{*}=B B^{+} A^{*} \text {. }
$$

Taking adjoints operators in both sides of the previous equality and multiplying on the right by $B$ gives

$$
A B B^{+} A^{+} A B=A B B^{+} B=A B .
$$

By ii), for every $A-$ a $\{1\}$-inverse of $A$, we have

$$
A B B^{+} A^{-} A B=A B B^{+} A^{+} A B=A B .
$$

From equations (1) and (2), it follows that for every $A^{-}$and for every $B^{-}\{1\}$ - inverses of $A$ and $B$ respectively, we have

$$
A B B^{-} A^{-} A B=A B
$$

which means that $B^{-} A^{-}$is a $\{1\}$-inverse of $A B$. The only if part: If equation (3) holds for every $A^{-}$and for every $B^{-}\{1\}$ - inverses of $A$ and $B$ respectively, then we have $A B B^{-} A^{+} A B=A B=A B B^{+} A^{-} A B$. It means that $A B B^{-} A^{+} A B$ and $A B B^{+} A^{-} A B$ are invariant under the choices of $B^{-}$and $A^{-}$. Thus both the two inclusions are satisfied.

Remark 1.1 If both $A$ and $B$ are two finite partial isometries. matrices $\left(A^{+}=A^{*}, B^{+}=B^{*}\right)$, then we have Greville's conditions for the reverse order law for the Moore-Penrose inverse of the product AB [2], page 176. 


\section{Characterization of the reverse order by the norm.}

The aim of this section is to characterize the condition of the reverse order under the Moore-Penrose inverse of the product of two closed range bounded linear operators in $H$ by the norms of these operators.

The following lemma is extended by Bouldin [3] and Izumino [9] from Greville's conditions on real or complex matrices to closed range bounded linear operators on Hilbert spaces,

Lemma 1.3 Let $A$ and $B$ be two closed range bounded linear operators on Hilbert space. Then $(A B)^{+}=B^{+} A^{+}$if and only if $\mathcal{R}\left(A^{*} A B\right) \subset \mathcal{R}(B)$ and $\mathcal{R}\left(B B^{*} A^{*}\right) \subset \mathcal{R}\left(A^{*}\right)$.

By using the second condition in lemma 3.1 , we have the following theorem:

Theorem 1.3 Let $A$ and $B$ be two closed range bounded linear operators on Hilbert space, then

$(A B)^{+}=B^{+} A^{+}$if and only if there exist positive numbers $k$ and $l$ such that $\left\|B^{*} A^{*} A\right\| \leq k\left\|B^{*}\right\|$ and

$\left\|A B B^{*}\right\| \leq l\|A\|$

Proof. By lemma 3.1, $\mathcal{R}\left(A^{*} A B\right) \subset \mathcal{R}(B)$ and $\mathcal{R}\left(B B^{*} A^{*}\right) \subset \mathcal{R}\left(A^{*}\right)$ if and only if

$$
\left\|B^{*} A^{*} A x\right\| \leq k\left\|B^{*} x\right\| k \text { and }\left\|A B B^{*} x\right\| \leq l\|A x\|
$$

for some $k$ and $l$ positive numbers and for all $x$ in $H$. Now, for any $y \in H$,

$y \neq 0$, we can take $x=\frac{1}{\|y\|}$, so $\|x\|=1$. Thus, we get the desired result.

Theorem 2.3 Let A and B be two closed range bounded linear operators on Hilbert space with finite rank.

Then, $(A B)^{+}=B^{+} A^{+}$if and only if $\left\|B^{*} A^{*} A\right\| \leq\left((r(B))^{\frac{1}{2}} k\left\|A^{*} A\right\|\right)\left\|B^{*}\right\|$ and $\left\|A B B^{*}\right\| \leq\left((r(A))^{\frac{1}{2}}\left\|B B^{*}\right\|\right)\|A\|$.

Proof. As $B B^{+}$and $A^{+} A$ are orthogonal projectors on $\mathcal{R}(B)$ and $\mathcal{R}\left(A^{*}\right)$, the inclusions in lemma 3.1 hold if and only if

$$
A^{*} A B=B B^{+}\left(A^{*} A B\right) B B^{*} A^{*}=A^{+} A\left(B B^{*} A^{*}\right)
$$

By taking the conjugate transpose of both sides in equations 5 , we have

$$
B^{*} A^{*} A=\left(B^{*} A^{*} A\right) B B^{+} A B B^{*}=\left(A B B^{*}\right) A^{+} A \text {. }
$$


Then for all $x \in H,\|x\|=1$, we have

$$
\begin{gathered}
\left\|B^{*} A^{*} A x\right\|=\left\|B^{*} A^{*} A B B^{+} x\right\| \leq\left\|B^{*}\right\|\left\|A^{*} A\right\|\left\|B B^{+}\right\|\|x\| \\
\left\|A B B^{*} x\right\|=\left\|A B B^{*} A^{+} A x\right\| \leq\|A\|\left\|B B^{*}\right\|\left\|A^{+} A\right\|\|x\| .
\end{gathered}
$$

As $B B^{+}$and $A^{+} A$ are orthogonal projectors, we have

By the same calculation, we have

$$
\left\|A^{+} A\right\|=\left(r\left(A^{+} A\right)\right)^{\frac{1}{2}}=(r(A))^{\frac{1}{2}} .
$$

Therefore, the inequalities in( 6) become:

$$
\left\|B^{*} A^{*} A\right\| \leq\left((r(B))^{\frac{1}{2}}\left\|A^{*} A\right\|\right)\left\|B^{*}\right\| \quad \text { and }
$$

Inversely, by putting $\quad k=\left(\left(r(B)^{\frac{1}{2}}\right)\|A\| A\right)$ and $l=\left((r(A))^{\frac{1}{2}}\left\|B B^{*}\right\|\right)$, in Theorem1.3, we have the converse.

The following example serves to show how characterizing range inclusion conditions by the norm majorization.

Example 1.3 $\quad A=\left(\begin{array}{ccc}-1 & 0 & 1 \\ 0 & 0 & -1 \\ 0 & 0 & 0\end{array}\right), B=A^{*} A=\left(\begin{array}{ccc}1 & 0 & -1 \\ 0 & 0 & 0 \\ -1 & 0 & 2\end{array}\right)=B^{*}$,

then Greville's conditions are satisfied;

$R\left(A^{*} A B\right)=R\left(B^{2}\right) \subset R(B), R\left(B B^{*} A^{*}\right)=R\left(B^{2} A^{*}\right)=R\left(\left(A^{*} A\right)^{2} A^{*}\right) \subset R\left(A^{*}\right)$,

Thus, $(A B)^{+}=B^{+} A^{+}$.

As $A^{*} A=\left(\begin{array}{ccc}1 & 0 & -1 \\ 0 & 0 & 0 \\ -1 & 0 & 2\end{array}\right) A^{*} A=\left(\begin{array}{ccc}1 & 0 & -1 \\ 0 & 0 & 0 \\ -1 & 0 & 2\end{array}\right)$ is self-adjoint, there exists an orthogonal matrix

$U=\left(\begin{array}{ccc}0 & \frac{1+\sqrt{5}}{\sqrt{10+2 \sqrt{5}}} & \frac{1-\sqrt{5}}{\sqrt{10-2 \sqrt{5}}} \\ 1 & 0 & 0 \\ 0 & \frac{\sqrt{2}}{\sqrt{5+\sqrt{5}}} & \frac{\sqrt{2}}{\sqrt{5-\sqrt{5}}}\end{array}\right) \quad$ such that $\quad B=A^{*} A=U\left(\begin{array}{ccc}0 & 0 & 0 \\ 0 & \frac{3-\sqrt{5}}{2} & 0 \\ 0 & 0 & \frac{3+\sqrt{5}}{2}\end{array}\right) U^{*}$ 
Now, we compute $\left\|B^{*} A^{*} A\right\|$ and $\left\|A B B^{*}\right\|$ :

$$
\begin{aligned}
& \left\|B^{*}\right\|=\left(\operatorname{tra}\left(B B^{*}\right)\right)^{\frac{1}{2}}=\left(\operatorname{tra}\left(B^{2}\right)\right)^{\frac{1}{2}}=\sqrt{7} . \\
& \left\|B B^{*}\right\|=\left(\operatorname{tra}\left(B B^{*}\right)^{2}\right)^{\frac{1}{2}}=\left(\operatorname{tra}\left(B^{4}\right)\right)^{\frac{1}{2}}=\sqrt{47 .} \\
& \left\|B^{*} A^{*} A\right\|=\left\|B^{*} B\right\|=\sqrt{47} \\
& \leq\left((r(B))^{\frac{1}{2}}\left\|A^{*} A\right\|\right)\left\|B^{*}\right\|=\sqrt{2}\left(\left\|B^{*}\right\|\right)^{2}=7 \sqrt{2} . \\
& \left\|A B B^{*}\right\| \leq\|A\|\left\|B B^{*}\right\|=\left(\operatorname{tra}\left(A^{*} A\right)\right)^{\frac{1}{2}} \sqrt{47}=\sqrt{3} \sqrt{47}=\sqrt{141} \\
& \leq\left((r(A))^{\frac{1}{2}}\left\|B B^{*}\right\|\right)\|A\|=\sqrt{2} \sqrt{141} .
\end{aligned}
$$

\section{References}

[1] R. B. Bapat, Linear Algebra and Linear Models, 2nd Edition, Springer (2000).

[2] A. Ben-Israel and T. N. E. Greville, Generalized inverses, theory and applications, Springer-Verlag NewYork, Inc (2003).

[3] R.H. Bouldin, The pseudo-inverse of a product, SIAM J. Appl. Math. 25 (1973) 489-495.

[4] S. R. Caradus, Generalized inverses and operator theory, Queen's paper in pure and applied mathematics, Queen's University, Kingston, Ontario,(1978).

[5] D. S. Djordjevi'c and N. C. Din 'ci'c, Reverse order law for the Moore-Penrose inverse, Journal of Mathematical Analysis and Applications, 361 (2010), 252-261.

[6] D. S. Djordjevi'c and V. Rako cevi'c, Lectures on Generalized Inverses, Faculty of Sciences and Mathematics, University of $\mathrm{Ni} \asymp \mathrm{s},(2008)$.

[7] R. G. Douglas, On majorization, factorization and range inclusion of operators on Hilbert spaces, AMS. Proc., $17, \mathrm{n} 2,(1966), 413-415$.

[8] M. R. Embry, Factorization of operators on Banach space, AMS. Proc., 38, n 3 (1973), 587-590.

[9] S. Izumino, The product of operators with closed range and an extension of the reverse order law, Tohoku Math. J. 34 (1982) 43-52.

[10] S. Karanasios An Introduction to Generalized Inverses of Operators on Banach and Hilbert Spaces, 2nd Summer School "Operator Theory" -Karlovasi, Athen, (2012), Part III.

[11] M.Z. Nashed, Generalized Inverses, Theory and Applications, Academic Press, NY (1976).

[12] H. Zekraoui and S. Guedjiba, Equivalent conditions for the reverse order property invariance under generalized inverses of matrices, AIP Conf. Proc. 1309, (2010), 927-932.

[13] H. Zekraoui, Propri’et'es Algebriques des Gk-Inverses des Matrices (in French), Batna, Algeria, (2011). 